\title{
Switching between dynamic states in intermediate-length Josephson junctions
}

Pagano, S.; Sørensen, Mads Peter; Parmentier, R. D.; Christiansen, Peter Leth; Skovgaard, Ove; Mygind, Jesper; Pedersen, Niels Falsig; Samuelsen, Mogens Rugholm

Published in:

Physical Review B

Link to article, DOI:

10.1103/PhysRevB.33.174

Publication date:

1986

Document Version

Publisher's PDF, also known as Version of record

Link back to DTU Orbit

Citation (APA):

Pagano, S., Sørensen, M. P., Parmentier, R. D., Christiansen, P. L., Skovgaard, O., Mygind, J., Pedersen, N. F., \& Samuelsen, M. R. (1986). Switching between dynamic states in intermediate-length Josephson junctions. Physical Review B, 33(1), 174-182. https://doi.org/10.1103/PhysRevB.33.174

\section{General rights}

Copyright and moral rights for the publications made accessible in the public portal are retained by the authors and/or other copyright owners and it is a condition of accessing publications that users recognise and abide by the legal requirements associated with these rights.

- Users may download and print one copy of any publication from the public portal for the purpose of private study or research.

- You may not further distribute the material or use it for any profit-making activity or commercial gain

- You may freely distribute the URL identifying the publication in the public portal 


\title{
Switching between dynamic states in intermediate-length Josephson junctions
}

\author{
S. Pagano," M. P. Soerensen, R. D. Parmentier, ${ }^{*}$ P. L. Christiansen, and O. Skovgaard \\ Laboratory of Applied Mathematical Physics, The Technical University of Denmark, DK-2800 Lyngby, Denmark \\ J. Mygind, N. F. Pedersen, and M. R. Samuelsen \\ Physics Laboratory I, The Technical University of Denmark, DK-2800 Lyngby, Denmark
}

(Received 15 July 1985)

\begin{abstract}
The appearance of zero-field steps (ZFS's) in the current-voltage characteristics of intermediatelength overlap-geometry Josephson tunnel junctions described by a perturbed sine-Gordon equation (PSGE) is associated with the growth of parametrically excited instabilities of the McCumber background curve (MCB). A linear stability analysis of a McCumber solution of the PSGE in the asymptotic linear region of the MCB and in the absence of magnetic field yields a Hill's equation which predicts how the number, locations, and widths of the instability regions depend on the junction parameters. A numerical integration of the PSGE in terms of truncated series of timedependent Fourier spatial modes verifies that the parametrically excited instabilities of the MCB evolve into the fluxon oscillations characteristic of the ZFS's. An approximate analysis of the Fourier mode equations in the presence of a small magnetic field yields a field-dependent Hill's equation which predicts that the major effect of such a field is to reduce the widths of the instability regions. Experimental measurements on $\mathrm{Nb}-\mathrm{Nb}_{x} \mathrm{O}_{y}-\mathrm{Pb}$ junctions of intermediate length, performed at different operating temperatures in order to vary the junction parameters and for various magnetic field values, verify the physical existence of switching from the MCB to the ZFS's. Good qualitative, and in many cases quantitative, agreement between analytic, numerical, and experimental results is obtained.
\end{abstract}

\section{INTRODUCTION}

The appearance of zero-field steps (ZFS's) in the current-voltage $(I-V)$ characteristics of long Josephson junctions results from fluxons propagating along the junction. This observation was first noted in a pioneering paper by Fulton and Dynes ${ }^{1}$ in 1973 . In the same paper Fulton and Dynes reported on experiments with a mechanical analog of a long, lightly damped junction consisting of a chain of elastically coupled plane pendula. In the regime of high mean voltage (angular velocity) they found a near-uniform rotation of the pendula, but with decreasing voltage they observed that this uniform mode of operation becomes unstable against spatial fluctuations, resulting in the creation of propagating fluxons or, alternatively, a switch to the zero-voltage state. In physical terms the near-uniform rotation corresponds to a junction which is biased on the McCumber curve.

In the present paper we report on analytic, numerical, and experimental results which elucidate in more detail the instability of the McCumber curve. The analytic work is based on a stability analysis of the perturbed sine-Gordon equation which describes the dynamics of the Josephson junction. In the case of zero magnetic field this equation is linearized around a solution which corresponds to a uniform rotation of the pendula in the mechanical analog. The result is a Hill's equation. The instability regions of this equation determine the instability intervals along the McCumber curve, the number of which gives the number of ZFS's that can be reached from the McCumber curve.

In the numerical work we use a method based on a simple extension of the multimode theory developed by Enpuku et al. ${ }^{2}$ which amounts to a consistent expansion of solutions of the perturbed sine-Gordon equation in truncated series of time-dependent Fourier spatial components. The time evolution of the Fourier coefficients is determined by direct numerical integration. The zeroorder Fourier coefficient corresponds to a near-uniform rotation which acts as a parametric driving force in the system. In the instability interval corresponding to the position of the $n$th zero-field step the zero-order Fourier coefficient excites predominantly the $n$th Fourier mode and gives rise to a spatial variation in the phase along the junction which evolves into the corresponding fluxon oscillation.

The effect of magnetic field is handled in an approximate way by means of a simplification of the multimode equations. After some manipulation, the problem is again reduced to a Hill's equation which now contains the magnetic field as a parameter.

The experimental samples studied are niobium-oxidelead junctions of overlap geometry. Experimental parameter values are adjusted by varying the sample temperature in a controlled way.

Comparison of the analytic, numerical, and experimental results yields an agreement that is at least qualitative and in many cases also quantitative. We have also observed some experimental phenomena, however, that are not contained in the model results. 


\section{MATHEMATICAL MODEL AND STABILITY ANALYSIS}

The mathematical model of the overlap Josephson junction is, in normalized form, the perturbed sine-Gordon equation $^{3}$

$$
\begin{aligned}
& \phi_{x x}-\phi_{t t}-\sin \phi=\alpha \phi_{t}-\beta \phi_{x x t}-\gamma, \\
& \phi_{x}(0, t)=\phi_{x}(l, t)=\eta .
\end{aligned}
$$

Here, $\phi(x, t)$ is the usual Josephson phase variable, $x$ is distance along the junction, normalized to the Josephson penetration length $\lambda_{j}$, and $t$ is time, normalized to the inverse of the Josephson plasma angular frequency $\omega_{0}$. The model contains five parameters: $\alpha, \beta, \gamma, l$, and $\eta$. The term in $\alpha$ represents shunt loss due to quasiparticles crossing the junction, the term in $\beta$ represents dissipation due to the surface resistance of the superconducting films, $\gamma$ is the uniform bias current normalized to the maximum zero-voltage Josephson current, $\eta$ represents the normalized external magnetic field, and the normalized length of the junction is denoted by $l$.

We first consider the case of homogeneous boundary conditions, i.e., $\eta=0$ in Eq. (1b). If $\alpha=\beta=\gamma=0$ the McCumber solution of Eqs. (1) is exactly ${ }^{4}$

$$
\phi=\phi_{0}(t)=2 \operatorname{am}[t / k ; k],
$$

where am is the Jacobian elliptic amplitude function ${ }^{5}$ of modulus $k$. For nonzero $\alpha, \beta$, and $\gamma$, we assume that Eq. (2) solves Eqs. (1) in the power-balance approximation. ${ }^{4}$ This yields the following expressions for the McCumber branch of the $I-V$ characteristic of the junction:

$$
\begin{aligned}
& \gamma=\frac{4 \alpha E(k)}{\pi k}, \\
& V=\left\langle\phi_{t}\right\rangle=\frac{\pi}{k K(k)},
\end{aligned}
$$

where $K(k)$ and $E(k)$ are, respectively, the complete elliptic integrals of first and second kinds. ${ }^{5}$

Following Burkov and Lifsic, ${ }^{6}$ we now express solutions of Eqs. (1) in the vicinity of the McCumber solution as

$$
\phi(x, t)=\phi_{0}(t)+\widetilde{\phi}(x, t),
$$

where $\phi_{0}$ is given by Eq. (2) together with the conditions of Eqs. (3), and $\widetilde{\phi}$ is a small perturbation of the form

$$
\widetilde{\phi}(x, t)=y(t) \exp (i b x)
$$

with $b$ constant. Inserting Eqs. (5) and (4) into Eqs. (1), we obtain an ordinary differential equation for $y(t)$ :

$$
\ddot{y}+\left(\alpha+\beta b^{2}\right) \dot{y}+\left\{b^{2}+\cos \left[\phi_{0}(t)\right]\right\} y=0,
$$

where

$$
b=n \pi / l, \quad n=0,1,2, \ldots
$$

and overdots denote derivatives with respect to $t$.

Equation (6a) is a damped Hill's equation; ${ }^{7}$ it may have unstable solutions in certain regions of its parameter space. In such regions a small initial disturbance will lead to a large response in the solution, giving rise to the onset of a solution with spatial structure, in contrast to the
McCumber solution. As will be seen in Sec. VI, such solutions evolve into the fluxon oscillations characteristic of ZFS's.

In the limit of small $k$, we may approximate Eq. (2) as ${ }^{5}$

$$
\phi_{0}(t)=\omega t+\frac{1}{\omega^{2}} \sin (\omega t),
$$

with

$$
\omega \equiv\left\langle\phi_{t}\right\rangle=\frac{\pi}{k K(k)} .
$$

This approximation is valid for the asymptotic linear portion of the McCumber curve, i.e., for $\omega \gtrsim 3$. The insertion of Eq. (7) into Eq. (6a) yields, after a simple calculation,

$$
\begin{aligned}
\ddot{y}+\left(\alpha+\beta b^{2}\right) \dot{y}+ & b^{2}-J_{1}\left(\omega^{-2}\right) \\
& +\sum_{m=1}^{\infty} a_{m} \cos (m \omega t) \mid y=0,
\end{aligned}
$$

where

$$
a_{m}=-\left\{\begin{array}{l}
2 m \omega^{2} J_{m}\left(\omega^{-2}\right), \quad m \text { odd } \\
2 J_{m}^{\prime}\left(\omega^{-2}\right), \quad m \text { even }
\end{array}\right.
$$

and $J_{m}$ is the Bessel function of first kind and $m$ th order, and $J_{m}^{\prime}$ denotes its derivative with respect to the argument.

Using the fact that the argument of all the Bessel functions in Eq. (9) is $1 / \omega^{2}$ and that by assumption $\omega \gtrsim 3$, we may approximate the Bessel functions as ${ }^{8}$

$$
J_{m}(x)=\frac{x^{m}}{2^{m} m !}-\frac{x^{m+2}}{4 \times 2^{m}[(m+1) !]} .
$$

Using this approximation, Eq. (9) may be rewritten as

$$
\ddot{y}+2 \epsilon \mu \dot{y}+\left(\delta+\sum_{m=1}^{\infty} \epsilon^{m} d_{m} \cos (m \tau)\right) y=0,
$$

where $\tau=\omega t, \epsilon=1 / \omega^{2}, \mu=(\omega / 2)\left(\alpha+\beta b^{2}\right)$,

$$
\delta=\left(\frac{1}{\omega^{2}}\right)\left(b^{2}-\frac{\omega^{-2}}{2}-\frac{\omega^{-6}}{16}\right),
$$

and overdots now denote derivatives with respect to $\tau$. The first few expansion coefficients are

$d_{1}=1-\frac{\omega^{-4}}{8}, \quad d_{2}=\frac{1}{2}-\frac{\omega^{-4}}{12}, d_{3}=\frac{1}{8}-\frac{\omega^{-4}}{128}$,

etc.

Following Nayfeh and Mook $^{7}$ we calculate the stability boundaries of Eq. (12) by means of a Lindstedt-Poincare perturbation expansion in the parameter $\epsilon$. Retaining terms up to second order, this calculation yields

$$
\begin{aligned}
b^{2}= & \left(\frac{\omega}{2}\right]^{2}+\frac{1}{2 \omega^{2}}\left[1-\frac{1}{8 \omega^{4}}\right) \\
& \pm \frac{1}{2}\left[\left[1-\frac{1}{8 \omega^{4}}\right]^{2}-\omega^{2}\left(\alpha+\beta b^{2}\right)\right]^{1 / 2}-\frac{1}{8 \omega^{2}}\left(1-\frac{1}{8 \omega^{4}}\right)^{2},
\end{aligned}
$$


with $b$ given by Eq. (6b). For given values of $\alpha, \beta, l$, and $n$, Eq. (14) gives two values for $\omega$, say $\omega_{+}$and $\omega_{-}$, which are the stability boundaries of Eq. (12), provided that the argument of the square root in Eq. (14) is positive. If this argument is negative, no instability region exists for the given parameter values. Using Eqs. (3) the voltagestability boundaries $\omega_{+}$and $\omega_{-}$can be translated into the corresponding current values, say $\gamma_{+}$and $\gamma_{-}$.

\section{MULTIMODE THEORY}

The linear-stability analysis presented in the preceding section provides estimates of the stability boundaries of the McCumber curve, but it cannot furnish the time evolution of an unstable solution. In order to follow the evolution of such an unstable solution, we consider a simple extension of the multimode theory developed by Enpuku et $a .^{2}$ The basic idea is to approximate solutions of Eqs. (1) in terms of a finite number of Fourier spatial modes whose amplitudes are unknown functions of time. This can be done with a reasonably small number of modes if two conditions are satisfied: (i) the spatial extent of a single fluxon is a sizable fraction of the length of the junction, and (ii) $\phi$ in Eqs. (1) can be expressed in terms of periodic, continuous, and smooth functions of $x$. Condition (i) will be satisfied if we limit attention to intermediate-length junctions, i.e., those having $1 \leq l \leq 5$. Condition (ii) can easily be satisfied in the following way: We first define a new function $\phi^{\prime}$ in the double $x$ interval $[-l, l]$ as

$$
\phi^{\prime}(x, t)=\left\{\begin{array}{l}
\phi(x, t), \quad 0 \leqq x \leqq l \\
\phi(-x, t),-l \leqq x \leqq 0
\end{array}\right.
$$

By construction $\phi^{\prime}$ is an even, continuous, periodic function of period $2 l$; however, from Eq. (1b), its spatial derivative is discontinuous at $x=0$ and $x= \pm l$ for $\eta \neq 0$. Therefore, we split $\phi^{\prime}$ into two parts, the first of which is an explicit function that satisfies Eq. (1b) and the second of which is now a smooth, even function, which, accordingly, may be reasonably approximated by a finite sum of low-order Fourier modes:

$$
\phi^{\prime}(x, t)=\eta|x|+\sum_{j=0}^{N} \theta_{j}(t) \cos \left(\frac{j \pi x}{l}\right) .
$$

Inserting Eq. (16) into Eqs. (1), we get, using the ortho- gonality properties of the cosine function together with the fact that $\phi^{\prime}$ is even,

$$
\begin{array}{r}
\ddot{\theta}_{0}+\alpha \dot{\theta}_{0}=\gamma-\frac{1}{l} \int_{x=0}^{l} \sin \left[\eta x+\sum_{j=0}^{N} \theta_{j} \cos \left(\frac{j \pi x}{l}\right)\right] d x \\
\ddot{\theta}_{m}+\left(\alpha+\beta \omega_{m}^{2}\right) \dot{\theta}_{m}+\omega_{m}^{2} \theta_{m} \\
=-\frac{2}{l} \int_{x=0}^{l} \sin \left[\eta x+\sum_{j=0}^{N} \theta_{j} \cos \left(\frac{j \pi x}{l}\right)\right] \\
\quad \times \cos \left(\frac{m \pi x}{l}\right) d x, m=1,2, \ldots, N
\end{array}
$$

with

$$
\omega_{m}=m \pi / l \text {. }
$$

This system was integrated numerically using the standard predictor-corrector routine DGEAR; ${ }^{9}$ the spatial integrals were evaluated by means of the fast-Fouriertransform routine FFTSC (Ref. 9) using $N$ function samples over the interval $[0, l]$ (corresponding to $2 N$ samples over one spatial period of $\phi^{\prime}$ ). The accuracy of the temporal integration was checked by varying the local error limit in DGEAR, and the influence of mode truncation by varying $N$.

\section{APPROXIMATE ANALYSIS OF MAGNETIC FIELD EFFECTS}

The multimode theory presented in the preceding section is valid for any value of the magnetic field $\eta$. Here we present an approximate treatment of this theory, valid for sufficiently small $\eta$, which reduces the problem of determining the effects of magnetic field to a simple analytic result similar to that presented in Sec. II. The approximation is based on assuming that the amplitudes of the spatial modes in Eqs. (17) are small, i.e.,

$$
\sum_{j=1}^{N}\left|\phi_{j}(t)\right| \ll 1 \text {. }
$$

Using this approximation we can calculate explicitly the integrals in Eqs. (17), obtaining

$$
\begin{aligned}
& \ddot{\theta}_{0}+\alpha \dot{\theta}_{0}-\gamma=-\left(c_{0,0}-\sum_{j=1}^{N} b_{j, 0} \theta_{j}\right) \sin \theta_{0}-\left(b_{0,0}-\sum_{j=1}^{N} c_{j, 0} \theta_{j}\right) \cos \theta_{0}, \\
& \ddot{\theta}_{m}+\left(\alpha+\omega_{m}^{2} \beta\right) \dot{\theta}_{m}+\omega_{m}^{2} \theta_{m}=-2\left(c_{0, m}-\sum_{j=1}^{N} b_{j, m} \theta_{j}\right) \sin \theta_{0}-2\left(b_{0, m}-\sum_{j=1}^{N} c_{j, m} \theta_{j}\right) \cos \theta_{0},
\end{aligned}
$$

with $m=1,2,3, \ldots, N$. Here,

$$
\begin{aligned}
& b_{j, m}=\frac{1}{4}\left[1-(-1)^{j+m} \cos (\eta l)\right]\left(\frac{1}{\eta l+\pi j+\pi m}+\frac{1}{\eta l+\pi j-\pi m}+\frac{1}{\eta l-\pi j+\pi m}+\frac{1}{\eta l-\pi j-\pi m}\right), \\
& c_{j, m}=\frac{1}{4}\left[(-1)^{j+m} \sin (\eta l)\right]\left(\frac{1}{\eta l+\pi j+\pi m}+\frac{1}{\eta l+\pi j-\pi m}+\frac{1}{\eta l-\pi j+\pi m}+\frac{1}{\eta l-\pi j-\pi m}\right) .
\end{aligned}
$$



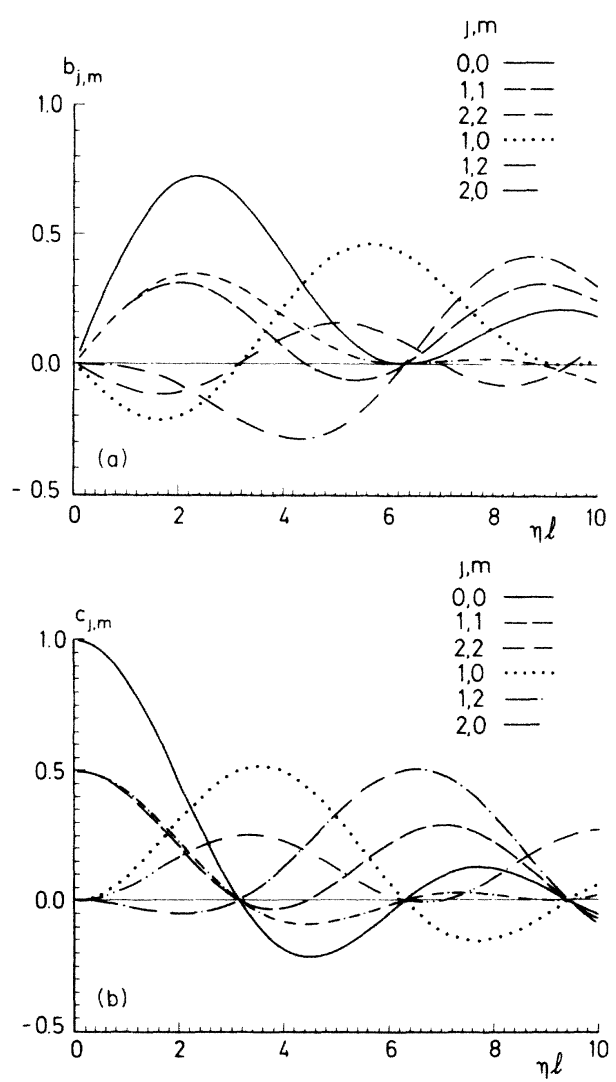

FIG. 1. Coefficients (a) $b_{j, m}$ and (b) $c_{j, m}$ of Eqs. (20). Note that $b_{j, m}=b_{m, j}$ and $c_{j, m}=c_{m, j}$.

In Fig. 1 we show the values of $b_{j, m}$ and $c_{j, m}$ for $j, m=0,1,2$ and $0 \leqq \eta l \leqq 10$.

Using again the approximation (18), together with the observation from Fig. 1 that the coefficients $b_{j, m}$ and $c_{j, m}$ are never larger in magnitude than 1, we may further approximate Eq. (19a) as

$$
\ddot{\theta}_{0}+\alpha \dot{\theta}_{0}-\gamma=-c_{0,0} \sin \theta_{0}-b_{0,0} \cos \theta_{0},
$$

which can be cast into the form

$$
\ddot{\theta}^{\prime}+\alpha^{\prime} \dot{\theta}^{\prime}-\gamma^{\prime}+\sin \theta^{\prime}=0,
$$

with

$$
\begin{aligned}
& a=\left(c_{0,0}^{2}+b_{0,0}^{2}\right)^{1 / 2}=\frac{\sin (\eta l / 2)}{\eta l / 2}, \\
& \delta=\arctan \left(b_{0,0} / c_{0,0}\right)=\eta l / 2, \\
& t^{\prime}=a^{1 / 2} t, \\
& \alpha^{\prime}=a^{-1 / 2} \alpha, \\
& \gamma^{\prime}=a^{-1} \gamma, \\
& \theta^{\prime}(t)=\theta_{0}(t)+\delta,
\end{aligned}
$$

where overdots now denote derivatives with respect to $t^{\prime}$. Equation (22a) has the same form as Eqs. (1) in the absence of spatial structure and in the absence of magnetic field, i.e., it describes a McCumber solution and consequently leads to

$$
\begin{aligned}
& \theta_{0}=2 \mathrm{am}\left[t a^{1 / 2} / k ; k\right]-\delta, \\
& \gamma=\frac{4 \alpha a^{1 / 2} E(k)}{\pi k}, \\
& \left\langle\dot{\theta}_{0 t}\right\rangle=\frac{\pi a^{1 / 2}}{k K(k)}
\end{aligned}
$$

If we now assume that the solution of Eqs. (19) contains only one dominant spatial mode, say the $m$ th (which is reasonable for a sufficiently short junction), Eqs. (19b) reduce to a single equation,

$$
\ddot{\theta}_{m}+\left(\alpha+\omega_{m}^{2} \beta\right) \dot{\theta}_{m}+\omega_{m}^{2} \theta_{m}-2\left(b_{m, m} \sin \theta_{0}-c_{m, m} \cos \theta_{0}\right) \theta_{m}=-2\left(c_{0, m} \sin \theta_{0}+b_{0, m} \cos \theta_{0}\right),
$$

which can be written as a Hill's equation with a forcing term

$$
\ddot{\theta}_{m}+\left(\alpha+\omega_{m}^{2} \beta\right) \dot{\theta}_{m}+\left[\omega_{m}^{2}+2 d \cos \left(\theta_{0}+\psi\right)\right] \theta_{m}=-2 \rho \sin \left(\theta_{0}+\xi\right)
$$

by defining

$$
\begin{aligned}
& d \equiv\left(c_{m, m}^{2}+b_{m, m}^{2}\right)^{1 / 2}=\frac{1}{2} \sin \left(\frac{\eta l}{2}\right)\left(\frac{1}{\eta l+2 \pi m}+\frac{1}{\eta l-2 \pi m}+\frac{2}{\eta l}\right), \\
& \psi \equiv \arctan \left(b_{m, m} / c_{m, m}\right)=\frac{\eta l}{2} \\
& \rho \equiv\left(c_{0, m}^{2}+b_{0, m}^{2}\right)^{1 / 2}=\sin \left(\frac{\eta l}{2}+\frac{\pi}{2} P_{m}\right)\left(\frac{1}{\eta l-\pi m}+\frac{1}{\eta l+\pi m}\right), \\
& \xi=\arctan \left(b_{0, m} / c_{0, m}\right)=\frac{\eta l}{2}+\frac{\pi}{2} P_{m},
\end{aligned}
$$

with $P_{m}=0,1$ for $m$ even,odd.

For small values of $\eta$ it is reasonable to assume that the instability regions of Eq. (25a) are the same as those of the associated homogeneous equation. ${ }^{10}$ Therefore, we restrict our attention to the homogeneous equation

$$
\ddot{\theta}_{m}+\left(\alpha+\omega_{m}^{2} \beta\right) \dot{\theta}_{m}+\left[\omega_{m}^{2}+2 d \cos \left(\theta_{0}+\frac{\eta l}{2}\right)\right] \theta_{m}=0 .
$$


We may perform now the same procedure as used in the $\eta=0$ case. As in that case, Eq. (26) may be cast into the form of Eq. (12), where now

$$
\tau=\omega t, \quad \epsilon=1 / \omega^{2}, \quad \mu=(\omega / 2)\left(\alpha+\beta \omega_{m}^{2}\right), \quad \delta=\omega^{-2}\left[\omega_{m}^{2}-2 d\left(\frac{\omega^{-2}}{2}-\frac{\omega^{-6}}{16}\right)\right] .
$$

The first few expansion coefficients are now given by

$$
d_{1}=2 d\left(1+\frac{\omega^{-4}}{8}\right), d_{2}=2 d\left(\frac{1}{2}-\frac{\omega^{-4}}{12}\right), d_{3}=2 d\left(\frac{1}{8}-\frac{\omega^{-4}}{128}\right)
$$

etc. Performing the same Lindstedt-Poincaré perturbation expansion used before, we obtain the following instability boundaries to second order in $\epsilon$ :

$$
\omega_{m}^{2}=\left(\frac{\omega}{2}\right)^{2}+\frac{d}{\omega^{2}}\left(1-\frac{1}{8 \omega^{4}}\right)-\frac{d^{2}}{2 \omega^{2}}\left[1-\frac{1}{8 \omega^{4}}\right]^{2} \pm\left[d^{2}\left[1-\frac{1}{8 \omega^{4}}\right)^{2}-\frac{\omega^{2}}{4}\left(\alpha+\beta \omega_{m}^{2}\right)^{2}\right]^{1 / 2}
$$

This expression assumes the form of Eq. (14) for $\eta=0$. For $\eta \neq 0$ but small it gives the dependence of the instability boundaries on the magnetic field.

\section{EXPERIMENTAL PROCEDURE}

Sample preparation and experimental technique have been described in previous publications. ${ }^{11}$ Several junctions have been investigated, all $\mathrm{Nb}-\mathrm{Nb}_{x} \mathrm{O}_{y}-\mathrm{Pb}$ overlap Josephson tunnel junctions of intermediate normalized length. The results reported here were obtained with a representative sample (S6-7/4), a junction of length $L=397 \mu \mathrm{m}$ and width $W=17.6 \mu \mathrm{m}$. Geometrically, the overlap of the junction was perfect to within $0.5 \mu \mathrm{m}$, the resolution of our optical microscope.

The substrate was mounted in a $1 \times 1$-in. $^{2}$ microstrip box and thermally anchored to a copper block containing two precision Ge thermometers and a small heating element. All the 50- $\mu \mathrm{m}$-diam wires leading to the Josephson junction, the thermometers, and the heating element were bifilarly wound and carefully thermally anchored to the copper block.

The microstrip box was included in a vacuum can immersed in a liquid-helium glass cryostat. A low-loss (inside gold-plated) rectangular stainless-steel waveguide connected the room-temperature $X$-band field-effecttransistor (FET) microwave receiver to a sma transition inside the vacuum can. The final part of the microwave system was a 20-cm-long, all- $\mathrm{Nb}, 0.085$-in. sma cable leading to a sma-to-stripline transition in the microstrip box.

A weak coupling to the microwave system was provided by an inverted microstrip antenna placed at a fixed distance of about $10 \mu \mathrm{m}$ from one end of the junction. The distance to the ground plane (nonsuperconducting) could be adjusted in situ by means of a cryogenic differential screw.

An extremely high stability of the three external bias parameters-temperature $T$, current $I_{\mathrm{dc}}$, and applied magnetic field $B_{\text {appl }}$-was essential and was verified by measuring the frequency $(\sim 10 \mathrm{GHz})$ and linewidth $(\sim 5$ $\mathrm{kHz}$ ) of the radiation emitted by the junction when biased on the first ZFS. Typical frequency-tuning rates of $\Delta v / \Delta T, \Delta v / \Delta I_{\mathrm{dc}}$, and $\Delta v / \Delta B_{\text {appl }}$ were $0.1 \mathrm{MHz} / \mathrm{mK}, 2$ $\mathrm{MHz} / \mu \mathrm{A}$, and $0.5 \mathrm{MHz} / \mu \mathrm{T}$, respectively.

The temperature of the helium bath was regulated with a manostat to within $\sim 1 \mathrm{mK}$. A temperature stabilization better than $10 \mu \mathrm{K}$ at $2.1<T<4.2 \mathrm{~K}$ could be maintained for minutes by adjusting the thermal time constant of the microstrip box by regulating the exchange-gas pressure in the vacuum can. All 50- $\mu \mathrm{m}$-diam wires were also thermally anchored to the vacuum can.

The dc-bias current was supplied either from a sweepable constant-current generator or from a current source based on reference mercury cells. The current was fed to the junction by a long, double $50-\mu \mathrm{m}$-diam wire, bifilarly wound transmission line, the hot end of which was thermostatted in order to minimize the influence of thermopowers. The dc voltage across the junction was measured using a similar transmission line. Both transmission lines were drawn inside thin-walled brass capillaries.

Input noise of either capacitive or inductive origin to the junction did not produce any observable frequency modulation or linewidth broadening of the emitted radiation. The noise of the dc amplifier used allowed us to resolve voltage-step structures less than $100 \mathrm{nV}$ on a fast (10-ms response) $X Y$ recorder.

The external magnetic field was produced by a coil wound onto the vacuum can and was applied in the plane of the junction and perpendicular to its long side. The magnetic shielding of the cryostat and the wires leading to the coil was sufficient to prevent magnetic noise from interfering with the measurements. This could be checked by reading the linewidth of the emitted radiation when biased alternatively in regions of the ZFS with $\Delta v / \Delta B_{\text {appl }}=0$ or $\Delta v / \Delta B_{\text {appl }} \neq 0$.

The critical-current density $J$, the Josephson penetration length $\lambda_{j}$, and the loss term $\alpha$ were determined from the dc $I-V$ characteristic (critical-current value, ZFS asymptotic voltage, and the slope of the McCumber curve at voltages corresponding to the ZFS studied) and from direct measurement of the plasma-resonance frequency. ${ }^{12}$ For the junction in question (sample S6-7/4), $J=26.2$ $\mathrm{A} / \mathrm{cm}^{2}, \lambda_{j}=91 \mu \mathrm{m}, l=L / \lambda_{j}=4.4$, and $\alpha=0.006$ at 4.2 $\mathrm{K}$. 


\section{RESULTS}

Figure 2 shows a portion of an $I-V$ characteristic calculated numerically from Eqs. (17) using the parameter values $\alpha=0.05, \beta=0.02, l=2$, and $\eta=0$. Both the McCumber background curve (MCB) and the first zerofield step (ZFS1) are evident. The inset shows in more detail the region where ZFS1 joins the McCumber curve. This region was calculated as follows: For $\eta=0$, the numerical growth of an instability requires the imposition of an inhomogeneous initial condition. Accordingly, for a given $\gamma$, a "pure" McCumber solution was launched and allowed to stabilize for 100 normalized time units, after which a small perturbation was added. In the instability region, i.e., for $\gamma_{-} \leqq \gamma \leqq \gamma_{+}$or $\omega_{-} \leqq \omega \leqq \omega_{+}$, the perturbation grows, causing the system to switch to ZFS1. Outside of the instability region the perturbation decays, and the system relaxes back to the McCumber curve. For $\eta \neq 0$ there is a coupling between the McCumber solution and the spatial modes through the boundary conditions, Eq. (1b); this allows the instability to develop also in the absence of an external perturbation.

Figure 3 shows the dynamics of switching in more detail. In this figure, obtained using the parameter values $\alpha=0.05, \beta=0.02, l=2, \eta=0$, and $\gamma=0.16$, a small perturbation has been added to the solution at a time prior to $t=500$. Figure 3(a) shows the behavior of $\dot{\theta}_{0}$ in Eqs. (17). Note from Eqs. (16) and (8) that $\left\langle\dot{\theta}_{0}\right\rangle=\left\langle\phi_{t}\right\rangle=\omega$. For $t \cong 500$, we see that $\omega \cong 3.20$. Between $t=500$ and 550 , a switching takes place, which, after a transient, settles into a state having $\omega \cong 2.85$. Figure 3 (b) shows the corresponding behavior of $\theta_{1}$ (the larger-amplitude oscillation) and $\theta_{2}$ (the smaller-amplitude oscillation) in Eqs. (17). From this figure it is evident that the switching seen in Fig. 3(a) is associated predominantly with an exponential growth of the first-order Fourier spatial component. In a similar way, Fig. 3(c) shows the behavior of $\theta_{3}$. Comparing Figs. $3(\mathrm{~b})$ and $3(\mathrm{c})$, we see that the amplitudes of the Fourier coefficients decrease rapidly with order number.

Using this numerical procedure, we find that the stability boundaries associated with ZFS1 for the parameter values used are, expressed in terms of bias current,

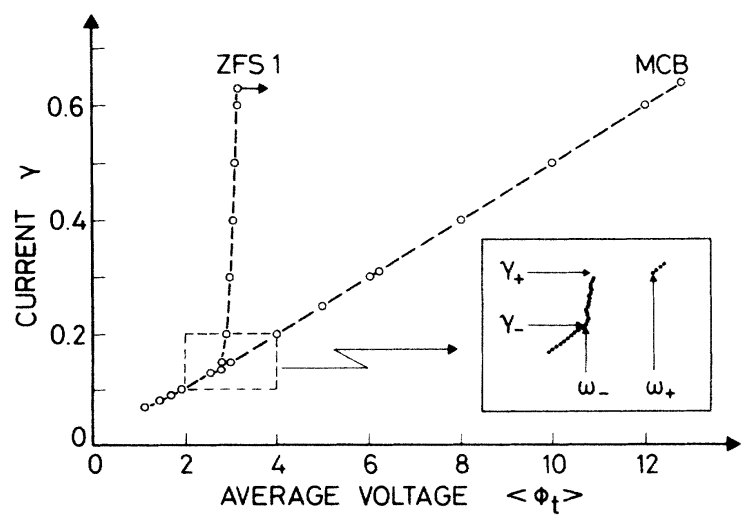

FIG. 2. $I-V$ characteristic calculation from Eqs. (17) using $\alpha=0.05, \beta=0.02, l=2$, and $\eta=0$, showing the McCumber background curve (MCB) and the first zero-field step (ZFS1). Inset shows detail of region where ZFS1 joins the MCB.
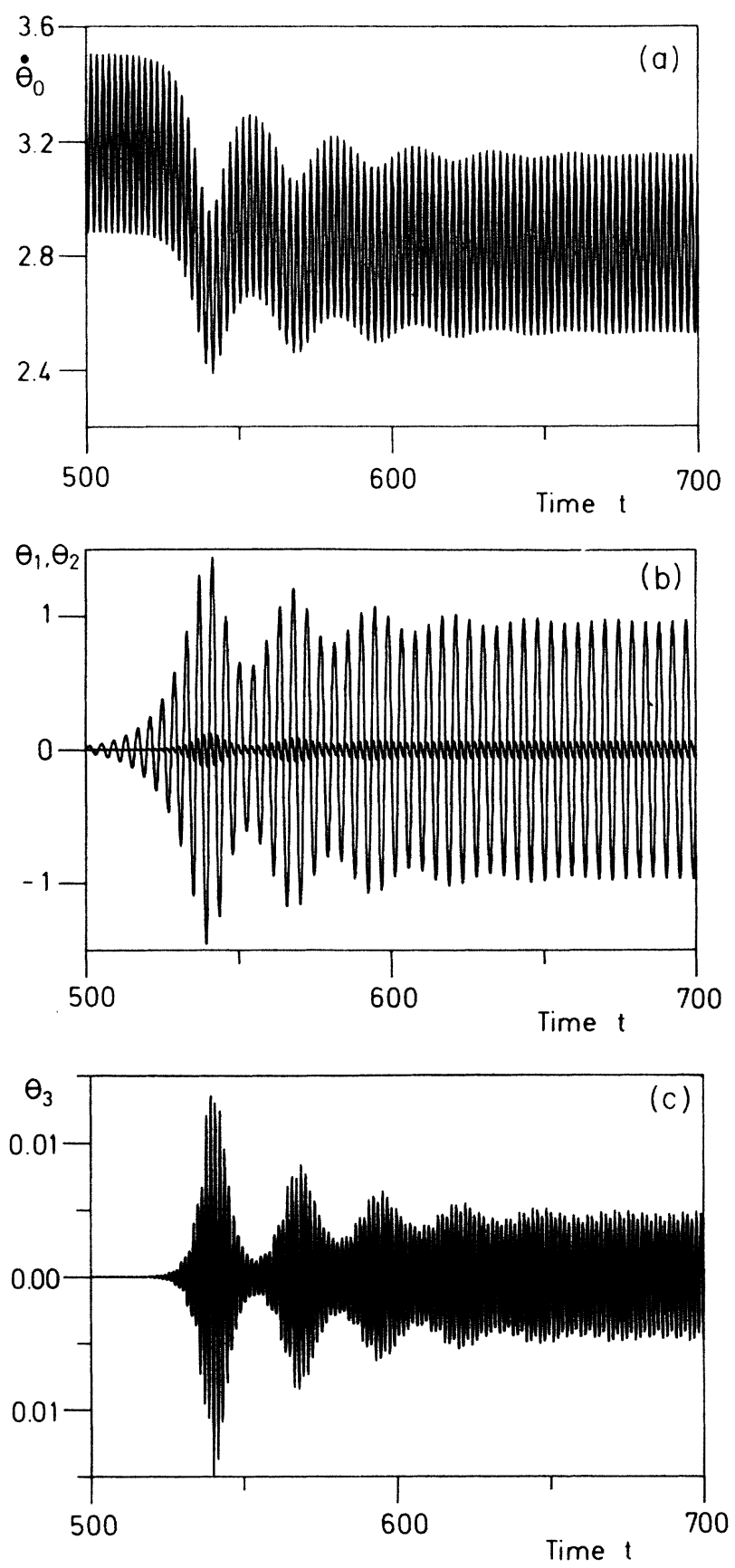

FIG. 3. Dynamics of switching from McCumber curve to ZFS1 calculated from Eqs. (17) by adding a small perturbation to the solution before $t=500$, in terms of Fourier coefficients: (a) $\dot{\theta}_{0}$, (b) $\theta_{1}$ (larger-amplitude oscillation) and $\theta_{2}$ (smalleramplitude oscillation), and (c) $\theta_{3}$.

$\gamma_{+}=0.1712 \pm 0.0005$ and $\gamma_{-}=0.1401 \pm 0.0001$. Inserting the same parameter values, together with $n=1$ in Eq. (6b), into Eq. (14) and (3), yields $\gamma_{+}=0.1711$ and $\gamma_{-}=0.1404$. Considering that this instability region occurs at the very lower end of the asymptotic linear region in the McCumber curve, for which the analysis of Sec. II was developed, the agreement is more than satisfactory.

Figure 4 shows the stability boundaries, now expressed in terms of average voltage, calculated from Eq. (14) with 


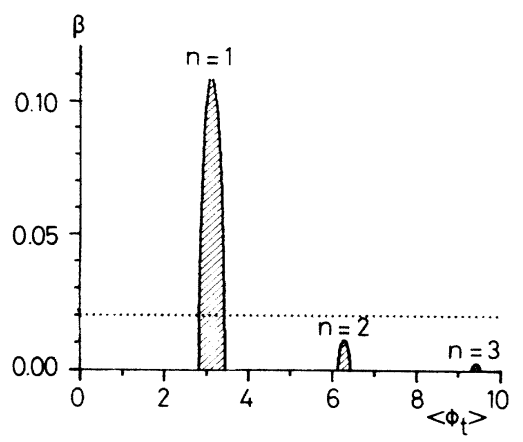

FIG. 4. Instability regions (shaded) in average voltage $\left\langle\phi_{t}\right\rangle$ calculated from Eq. (14) for ZFS1, ZFS2, and ZFS3 for different $\beta$-loss values using $\alpha=0.05$ and $l=2$. Dotted line corresponds to $\beta=0.02$ used in multimode theory.

$\alpha=0.05$ and $l=2$, for different values of $\beta$. The dotted line corresponds to the value $\beta=0.02$ used above. For these parameter values there is only one instability region in the McCumber curve; however, by lowering $\beta$ it is possible to have as many as six such regions (the three lowest of which are shown in Fig. 4). This result helps to clarify a long-standing experimental question, i.e., what determines the number of ZFS's that may be observed in the $I-V$ characteristic of a given junction?

This situation is illustrated in more detail for ZFS1 in Fig. 5(a) and for ZFS2 in Fig. 5(b). In these figures the solid curves are calculated from Eq. (28) and translated into bias current through Eqs. (3) (this is done to facilitate comparisons with the numerical results, in which $\gamma$ is a direct control parameter). The curves labeled 1 corresponds to $\eta=0$ and those labeled 2 to $\eta=0.8$. As is evi-
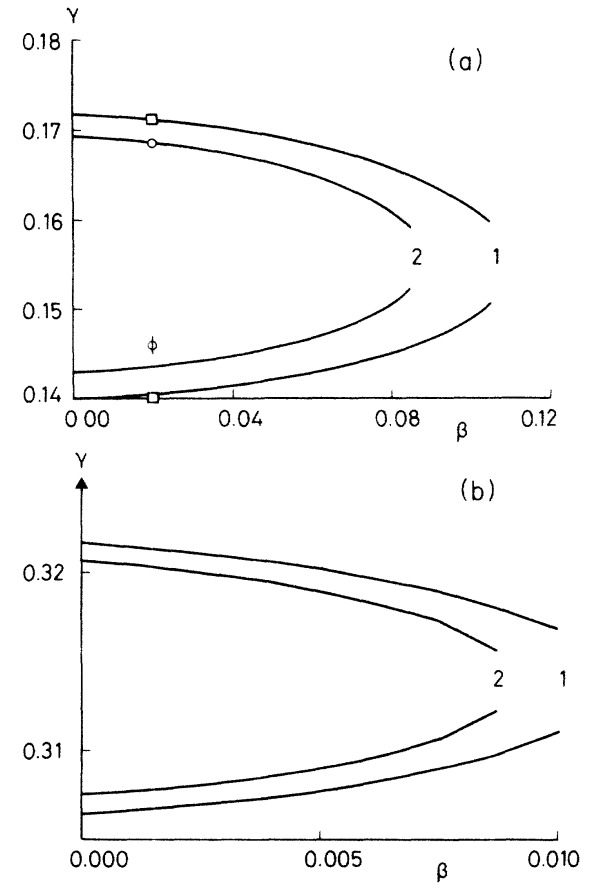

FIG. 5. Stability boundaries in current $\gamma$ as a function of $\beta$ loss calculated from Eq. (28) and Eqs. (3) for (a) ZFS1 and (b) ZFS2, for $\eta=0$ (curves 1) and $\eta=0.8$ (curves 2) using $\alpha=0.05$ and $l=2$. Squares and circles in (a) are calculated from Eqs. (17) for $\eta=0$ and 0.8 , respectively. dent from Fig. 5, the main effect of a (small) magnetic field is to reduce the width of the instability regions. The points indicated by squares and circles in Fig. 5(a) are calculated from Eqs. (17); the squares correspond to $\eta=0$ and the circles to $\eta=0.8$. The error bar on the lower circle reflects the fact that for $\eta \neq 0$ both the ZFS states and the McCumber states contain significant amounts of Fourier components, thus rendering a clear distinction between the two states somewhat difficult at the lower end of an instability region. This distinction is much sharper for the other multimode points in Fig. 5(a), and the relative error bars are contained within the dimensions of the symbols.

The dynamic state into which the system evolves after the switching shown in Fig. 3 is the fluxon oscillation state associated with ZFS1. This fact may be clearly established by comparing the results of the multimode theory, Eqs. (17), with those of the direct simulation solution of Eqs. (1) reported in Ref. 13. Figure 6 shows such a comparison. In this figure, the solid curve is the function $\phi_{t}(0, t)$, which is the voltage at the $x=0$ end of the junction, as obtained by direct simulation. The arrival of fluxons at the junction end is clearly apparent. The dashed curve is the same function as reconstructed from Eq. (16) using $N=3$. The bias value used in Fig. 6 is $\gamma=0.60$, i.e., near the top of ZFS1. For smaller values of $\gamma$ the agreement is even better, and the two curves are practically indistinguishable.

Figure 7(a) shows a portion of the $I-V$ characteristic of the experimental sample S6-7/4 measured at a temperature somewhat below the transition temperature of the lead counterelectrode and in zero magnetic field. The dashed arrows indicate switching from the zero-voltage current and from the first two ZFS's to the gap state. Figure $7(b)$ is the same characteristic with a $10 \times$ magnification of the current scale. The dotted lines in Fig. 7 indicate switching from higher-voltage to lower-voltage states. Clearly evident in Fig. 7(b) is a switching from the bottom of ZFS1 to the zero-voltage state. This may be due to a direct instability of the ZFS's toward the zero-voltage state $^{14}$ rather than being connected with an instability of the McCumber curve; such a mechanism is not contained in our present model.

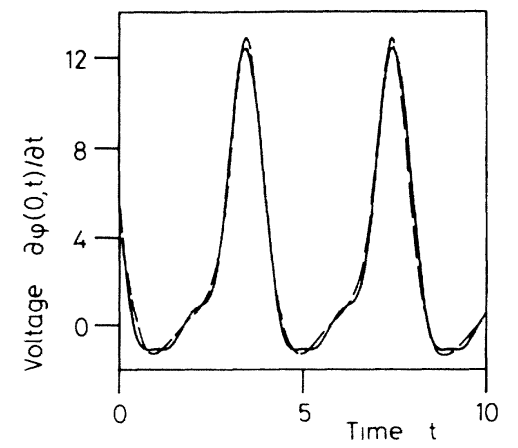

FIG. 6. Time evolution of voltage at $x=0$ junction end as obtained by direct numerical simulation of Eqs. (1) (solid curve) and as reconstructed from Eq. (16) using $N=3$ (dashed curve) for parameter values $\alpha=0.05, \beta=0.02, l=2, \quad \eta=0$, and $\gamma=0.60$. 


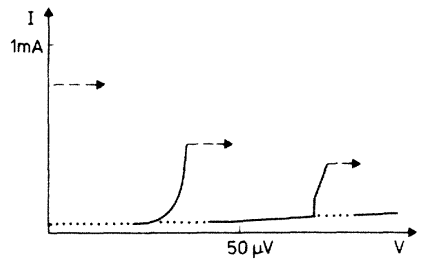

(a)

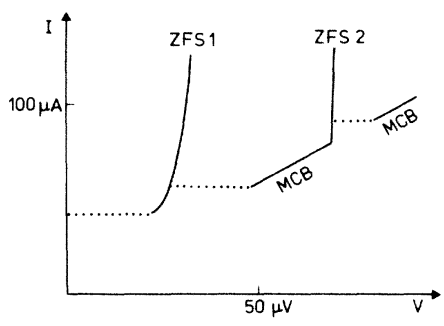

(b)

FIG. 7. (a) Detail of the $I-V$ characteristic of experimental sample S6-7/4 measured at a temperature slightly below the transition temperature of the lead counterelectrode and in zero magnetic field. Arrows indicate switching to the gap state. (b) Same characteristic with $10 \times$-magnified current scale. Dotted lines indicate switching from higher-voltage to lower-voltage states.

The characteristics shown in Fig. 7 correspond to a normalized junction length of about $l=3.2$ and an $\alpha$-loss term, estimated from the slope of the McCumber curve, of about $\alpha=0.03$. The experimental determination of the $\beta$-loss term is subject to rather large uncertainties, and so we have treated $\beta$ as an adjustable parameter in what follows. An essential feature of the experimental procedure is that the parameter values for a given junction can be "tuned" experimentally by varying the operating temperature.

Figure 8(a) shows a comparison between the experimentally determined stability boundaries (circles) in a magnetic field associated with ZFS1 and those obtained from Eq. (28), shown as solid lines. Experimental values of voltage and magnetic field were translated into normalized terms using the formulas $\left\langle\phi_{t}\right\rangle=V / \Phi_{0} f_{p}$ and $\eta=2 \pi \phi_{\text {ext }} / \Phi_{0} l$, where $V$ is the physical voltage, $\Phi_{0}$ is the magnetic flux quantum, $\phi_{\text {ext }}$ is the applied magnetic flux threading the junction, and $f_{p}$ is the plasma frequency. ${ }^{12}$ The experimental data were taken at a temperature for which $\alpha=0.026$ and $l=3.16$, and these same parameter values were used in Eq. (28). The $\beta$ value used in Eq. (28) was varied between 0 and 0.07 ; the effect of this variation is indicated by the slight thickening of the curves in Fig. 8(a). The agreement between the experimental and theoretical values for $\omega_{+}$is reasonable. The large discrepancy for the $\omega_{-}$branch may be due either to the fact that here $\omega$ lies considerably below the lower limit of the asymptotic linear region of the McCumber curve $(\omega \cong 3)$, or to the fact that a switching mechanism not described by the model is involved, as mentioned above.

Figure 8(b) shows a similar comparison for ZFS2, using the same parameter values (same temperature) as in Fig. 8(a). The $\beta$ value used in Eq. (28) was varied between 0

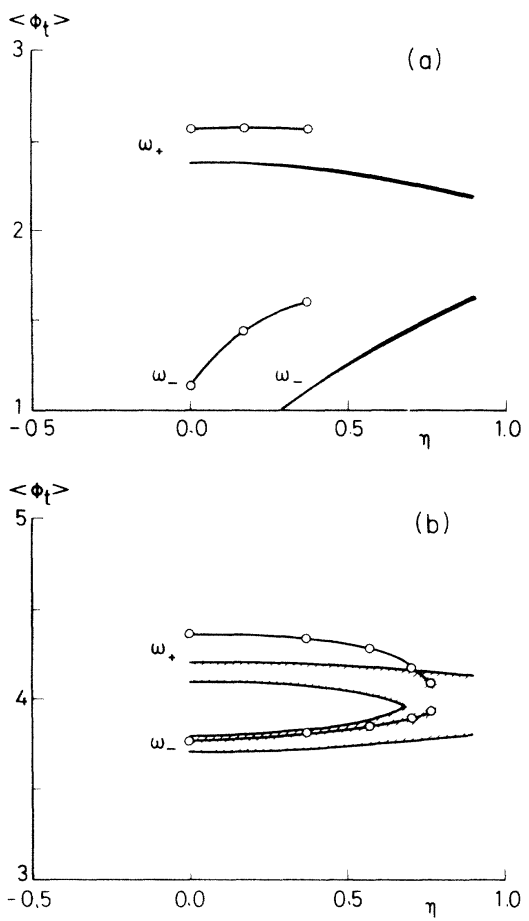

FIG. 8. Stability boundaries in average voltage $\left\langle\phi_{t}\right\rangle$ as a function of magnetic field $\eta$ measured experimentally (circles) and calculated from Eq. (28) (solid curves) for (a) ZFS1 and (b) ZFS2. Fixed parameter values: $\alpha=0.026$ and $l=3.16$. $0 \leqq \beta \leqq 0.07$ in (a) and $0 \leqq \beta \leqq 0.05$ in (b), giving rise to the shaded regions between the solid curves.

and 0.05 ; the effect of this variation is indicated by the shaded regions in Fig. 8(b). The agreement obtained here for the behavior of the $\omega_{-}$branch is much better than that of Fig. 8(a). The reason for this fact may be that there is no direct switching from ZFS2 to the zero-voltage state as there is for ZFS1, as can be seen in Fig. $7(\mathrm{~b})$, or that here $\omega_{-}>3$.

Figure 9 shows a similar result for ZFS1 at a higher temperature, for which $\alpha=0.043$ and $l=2.56$, and for a

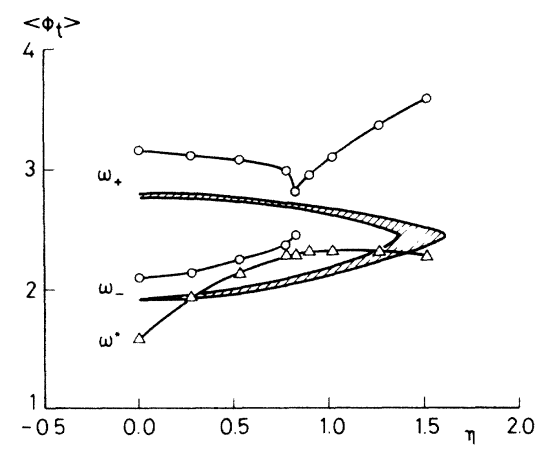

FIG. 9. Stability boundaries in average voltage $\left\langle\phi_{t}\right\rangle$ as a function of magnetic field $\eta$ measured experimentally (circles) and calculated from Eq. (28) (solid curves) for ZFS1 using fixed parameter values $\alpha=0.043$ and $l=2.56$. $\beta$ in Eq. (28) is varied in the range $0 \leqq \beta \leqq 0.07$, giving rise to the shaded regions between the solid curves. Triangles indicate experimental stability boundary $\omega^{*}$ of the stable piece of the McCumber curve that appears below the bottom of ZFS1. 
larger range of magnetic field. As before, circles are experimental points and solid lines are calculated from Eq. (28). Also, as before, the shaded regions between the solid curves represent the effect of varying $\beta$ between 0 and 0.07. Two new phenomena are represented in Fig. 9. The first is that at a field value of about $\eta=0.8$, there is an abrupt change in the behavior of the experimental $\omega_{+}$ branch. Physically, this corresponds to the disappearances of ZFS1 with increasing field and the growth of the second Fiske step (FS2) at approximately the same voltage. $^{15}$ The perturbation theory result derived in Sec. IV is approximately valid only for sufficiently small values of $\eta$; it cannot be expected to hold for larger field values. The second new experimental phenomenon is the appearance of a stable portion of the McCumber curve below the bottom of ZFS1 at this temperature. In Fig. 9 the lower curve indicated by circles is, as before, the bottom of ZFS1, and the curve indicated by triangles is the lower stability boundary $\left(\omega^{*}\right)$ of this new piece of stable McCumber curve.

We note from Figs. 8 and 9 that the experimental stability boundaries are systematically higher than the corresponding theoretical ones. The reason for this might be that the asymptotic voltage of the ZFS's corresponds to normalized limiting velocities of the fluxons less than 1 , as discussed by Scheuermann and Chi. ${ }^{16}$ This would lead to a calculated value of the normalized length $l$ larger than the real value and, correspondingly, to a shift of the theoretical voltage positions on the normalized voltage scale to lower values since, in normalized terms, $l\left\langle\Phi_{t}\right\rangle=2 \pi$ for ZFS 1 .

\section{CONCLUSIONS}

A linear-stability analysis of the perturbed sine-Gordon equation which describes the dynamics of Josephson tunnel junctions indicates that the mechanism that determines the experimental observation of ZFS's may be described in terms of the growth of parametrically excited instabilities of the McCumber curve. This analysis gives good agreement with both numerical and experimental results in the asymptotic linear region of the McCumber curve and for sufficiently small values of the applied magnetic field. It would be useful to extend the analysis to the region of the McCumber curve below the asymptotic linear region in order to be able to study low-order steps in longer junctions.

Numerical integration of the multimode equations verifies that the parametrically excited instabilities evolve into fluxon oscillations. The multimode approach is a useful alternative to the direct numerical simulation of Eqs. (1) inasmuch as it gives reasonably reliable results at a considerably reduced computing cost. It should be remembered, however, that truncated mode expansions can be expected to give reliable results only when the dynamic states in question are reasonably smooth. Here, as elsewhere, the study of phenomena such as subharmonic generation and chaos will presumably require the use of different tools.

\section{ACKNOWLEDGMENTS}

We are grateful to S. E. Burkov for illuminating discussions regarding the stability analysis. We gratefully acknowledge financial support from the Danish Natural Science Research Council and from the European Research Office of the United States Army through Contract No. DAJA-37-82-C-0057. One of us (S.P.) thanks the Fondazione Angelo della Riccia (Italy) for partial support for the period during which this work was performed. Another of us (R.D.P.) was supported in part by the Gruppo Nazionale della Struttura di Materia, Consiglio Nazionale della Ricerche-CISM, Ministero della Publica Istruzione (GNSM/CNR-CISM/MPI) (Italy) and in part by the Thomas B. Thriges Fond (Denmark).
"Permanent address: Dipartimento di Fisica, Università degli Studi di Salerno, I-84100 Salerno, Italy.

${ }^{1}$ T. A. Fulton and R. C. Dynes, Solid State Commun. 12, 57 (1973).

${ }^{2}$ K. Enpuku, K. Yoshida, and F. Irie, J. Appl. Phys. 52, 344 (1981).

${ }^{3}$ P. S. Lomdahl, O. H. Soerensen, and P. L. Christiansen, Phys. Rev. B 25, 5737 (1982).

${ }^{4}$ R. D. Parmentier, in Solitons in Action, edited by K. Lonngren and A. Scott (Academic, New York, 1978), pp. 173-199.

${ }^{5}$ P. F. Byrd and M. D. Friedman, Handbook of Elliptic Integrals for Engineers and Physicists (Springer, Berlin, 1954).

${ }^{6}$ S. E. Burkov and A. E. Lifsic, Wave Motion 5, 197 (1983).

${ }^{7}$ A. H. Nayfeh and D. T. Mook, Nonlinear Oscillations (WileyInterscience, New York, 1979), Chap. 5.

${ }^{8} \mathrm{M}$. Abramowitz and I. Stegun, Handbook of Mathematical Functions, 9th ed. (Dover, New York, 1970), Eq. 9.1.10.

${ }^{9}$ IMSL, Inc. (Houston, Texas), International Mathematical and Statistical Library (Edition 9), 1982 (unpublished).
${ }^{10}$ C. S. Hsu and W. H. Cheng, J. Appl. Mech. ASME 41, 371 (1974).

${ }^{11}$ B. Dueholm, O. A. Levring, J. Mygind, N. F. Pedersen, O. H. Soerensen, and M. Cirillo, Phys. Rev. Lett. 49, 1093 (1982).

$12 \mathrm{~J}$. Bindslev Hansen, J. Mygind, S. Pagano, M. R. Samuelsen, and H. Svensmark, in Proceedings of the International Conference on Tunneling at Low Temperatures, Heverlee, Belgium, 25-30 August 1985 (unpublished).

${ }^{13}$ M. P. Soerensen, R. D. Parmentier, P. L. Christiansen, O. Skovgaard, B. Dueholm, E. Joergensen, V. P. Koshelets, O. A. Levring, R. Monaco, J. Mygind, N. F. Pedersen, and M. R. Samuelsen, Phys. Rev. B 30, 2640 (1984).

${ }^{14}$ N. F. Pedersen and D. Welner, Phys. Rev. B 29, 2551 (1984).

${ }^{15}$ M. Cirillo, A. M. Cucolo, S. Pace, and B. Savo, J. Low Temp. Phys. 54, 489 (1984).

${ }^{16} \mathrm{M}$. Scheuermann and C. C. Chi, in Proceedings of the Third International Conference on Superconducting Quantum Devices, West Berlin, 25-28 June 1985, Conference Digest, p. 170 (unpublished). 